\title{
A SECOND-ORDER EXTENSION OF TV REGULARIZATION FOR IMAGE DEBLURRING
}

\author{
Zafer Dogan, Stamatios Lefkimmiatis, Aurélien Bourquard, and Michael Unser \\ Biomedical Imaging Group (BIG), EPFL, CH-1015 Lausanne, Switzerland
}

\begin{abstract}
In this paper, we propose a novel second-order regularizer based on the maximum response of the second-order directional derivative, assuming that the image under consideration belongs to the class of piecewise-linear signals. Compared to total-variation regularization that preserves edges but transforms piecewise-smooth regions into piecewise-constant regions, the proposed model is able to restore piecewise-linear regions and finer details. Deconvolution experiments demonstrate the performance of our approach in terms of the quality of reconstruction.
\end{abstract}

Index Terms - Image deconvolution, Linear inverse problems, Higher-order regularization.

\section{INTRODUCTION}

Every imaging system causes an inevitable degradation of quality during acquisition. In that regard, the goal of deblurring is to improve the contrast and the perceived resolution of the degraded images. A common approach is to express the deblurring problem in a variational framework, using a regularization term to model the a priori knowledge about the input [1].

Total variation (TV) regularization has been introduced by Rudin et al. in 1992 [2] as a denoising algorithm. The principle of TV states that signals with excessive and possibly spurious details have high total variation. Reducing the total variation of the image while keeping the solution close to the original signal via some data-fidelity term removes noise whilst preserving the sharpness of edges. TV-based regularization is particularly well-suited to image restoration in certain cases. The underlying assumption of TV is that images under consideration belong to the class of piecewise-constant signals. Hence, the recovered images resulting from the application of this model in the presence of noise are subject to the so-called staircase effect. Therefore, the use of TV may generate undesirable artifacts and compromise the quality of the recovered image.

To overcome this problem, researchers have proposed using second (or higher)-order regularization methods. The related work can be categorized into two groups. The first one

This work was supported (in part) by the Hasler Foundation and the Indo-Swiss Joint Research Program. tries to reduce the staircase effect introduced by TV using jointly the total-variation regularization and another regularization term based on some higher-order derivatives $[3,4]$. The second category considers regularization schemes solely based on higher-order derivatives [5, 6].

In the next sections, our contribution is to propose a new second-order regularization functional that falls in that second category, and that is derived as an extension of TV. We propose a cost-minimization technique in Sec. 5, and provide experimental results in Sec. 6.

\section{PROBLEM FORMULATION}

Following a variational approach, we propose to solve the general problem

$$
\hat{f}=\arg \min _{f} J\{f\}
$$

with

$$
J\{f\}=\frac{1}{2}\|y-\mathrm{A}\{f\}\|_{2}^{2}+\tau J_{\text {reg }}\{f\},
$$

where A is a convolution operator, $y$ is the noisy and blurred observation, and $f$ is the signal we are recovering. The generic regularization term $J_{\text {reg }}$ is weighted by a constant $\tau$. In this paper, we consider $J_{\text {reg }}$ within the class of convex regularizers that involve some norm of a linear differential operator L of first or higher order, in a given number of dimensions.

Assuming suitable differentiability properties on $f$, the $2 \mathrm{D}$ version of TV amounts to minimizing the maximummagnitude directional derivative at each position. The firstorder directional derivative of $f$ along the unit vector $\mathbf{u}_{\theta}=$ $(\cos \theta, \sin \theta)$ is $\mathrm{D}_{\mathbf{u}_{\theta}} f(\mathbf{x})=\langle\mathbf{u}, \nabla f(\mathbf{x})\rangle$, and its maximum angular magnitude is $\max _{\theta}\left|\mathrm{D}_{\mathbf{u}_{\theta}} f(\mathbf{x})\right|=\|\nabla f(\mathbf{x})\|$. Hence, the TV regularization term can be reformulated as

$$
J_{T V}\{f\}=\left\|\max _{\theta}\left|\mathrm{D}_{\mathbf{u}_{\theta}} f(\mathbf{x})\right|\right\|_{1} .
$$

From that perspective, our new second-order regularizer is meant to extend this directional-derivative-maximization principle to the second-order case, while keeping the beneficial properties of the $\mathrm{L}_{1}$-norm stated in [2].

In TV, signals are assumed to be piecewise-constant, while our approach implicitly adopts a piecewise-linear 


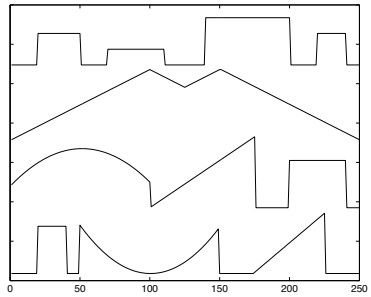

(a) Original

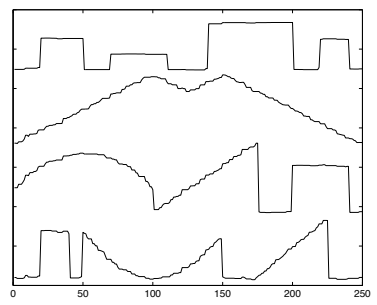

(c) Denoised with TV

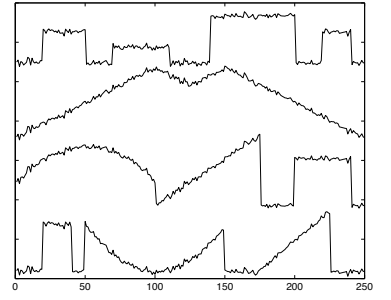

(b) Noisy

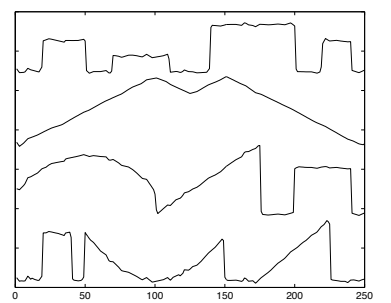

(d) Denoised with our model
Fig. 1. Denoising results on 1D signals

model. Note that, from an approximation viewpoint, the decomposition of signals into piecewise-linear functions yields a lesser approximation error than decomposing them into piecewise-constant functions. Therefore, assuming that the signals under consideration can be better decomposed into piecewise-linear functions, we expect second-order regularization to be more accurate in terms of restoration quality.

\section{THE 1D MODEL}

In this section, we illustrate and motivate the use of higherorder information for regularization, confronting our model with TV. To better understand the essential characteristics of each method, we first consider the 1D case where both regularizers degenerate to the $\mathrm{L}_{1}$-norm of the first and second derivatives, respectively. In order to illustrate the behavior of the new regularizer, we consider comparisons in a pure denoising setting (i.e., the blurring operator being the identity). The result is thus not affected by smoothing and is easier to evaluate.

In this denoising experiment, we consider four types of synthetic signals ( piecewise-constant, piecewise-linear, mixed, and piecewise-parabolic). The original signals are degraded by additive zero-mean Gaussian noise of standard deviation $\sigma=2$.

In Fig. 1, we observe the edge-preserving property of TV as well as its characteristic staircase artifacts. We also notice that, in the case of TV, nonconstant regions are broken to smaller piecewise-constant ones. In contrast, our model reconstructs piecewise-linear regions more accurately without staircase artifacts, while still preserving sharp edges. Hence, the second-order regularization is an interesting alternative to $\mathrm{TV}$, especially when dealing with piecewise-smooth signals.

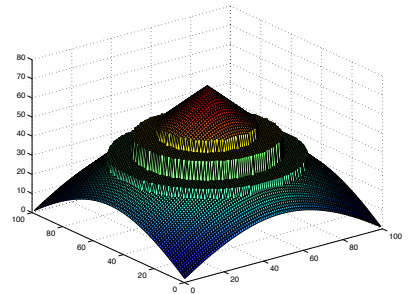

(a) Original image

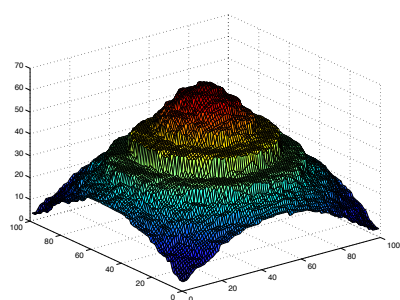

(c) TV solution

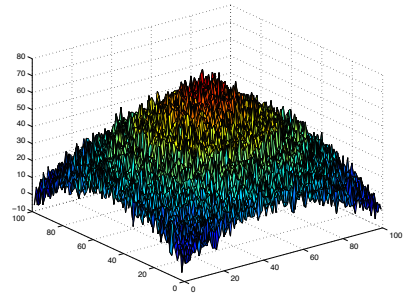

(b) Degraded image

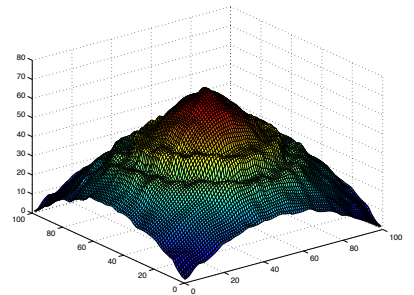

(d) Our solution
Fig. 2. Intensity plots on a 2D synthetic example

\section{THE 2D MODEL}

In this section, our second-order regularization term is derived for the $2 \mathrm{D}$ case, based on the minimization of the maximum magnitude of second-order directional derivatives at each coordinate. Assuming $f(\mathbf{x})$ to be twice-differentiable, its directional second derivative along the unit vector $\mathbf{u}_{\theta}=$ $(\cos \theta, \sin \theta)$ is $\mathrm{D}_{\mathbf{u}_{\theta}}^{2} f(\mathbf{x})=\mathbf{u}_{\theta}^{T}(\mathrm{H}\{f\}(\mathbf{x})) \mathbf{u}_{\theta}$, where $\mathrm{H}$ is the $2 \times 2$ Hessian operator. The maximum-magnitude response is then $\max \left|\mathrm{D}_{\mathbf{u}_{\theta}}^{2} f(\mathbf{x})\right|=\max \left(\left|\lambda^{ \pm}(\mathbf{x})\right|\right)$, where $\lambda^{ \pm}$ corresponds to

$$
\lambda^{ \pm}=\frac{1}{2}\left[\Delta f \pm \sqrt{\left(\frac{\partial^{2} f}{\partial x_{1}^{2}}-\frac{\partial^{2} f}{\partial x_{2}^{2}}\right)^{2}+\left(\frac{2 \partial^{2} f}{\partial x_{1} \partial x_{2}}\right)^{2}}\right]
$$

In other words, the maximum magnitude of the directional second derivative at a given image coordinate corresponds to the maximum absolute eigenvalue (or, equivalently in that case, the spectral radius) of the corresponding Hessian matrix. Hence, the proposed regularization method is convex and can be written in the more concise form

$$
J_{\text {new }}\{f\}=\|\rho(H\{f\}(\mathbf{x}))\|_{1},
$$

where $\rho$ takes the spectral radius of its matrix argument.

As in the 1D case, we want to compare the proposed 2D regularizer with TV. This is done by means of a synthetic image that includes piecewise-linear as well as piecewiseconstant intensity regions. This experiment is once more carried out in a denoising setting, with Gaussian noise of $\sigma=4$.

We represent the oracle, degraded, and restored signals in Fig. 2. In order to better visualize the results, we display the corresponding intensities as three-dimensional plots. As a 
matter of fact, 2D TV restoration does obviously not discriminate between sudden intensity jumps and smooth transitions. As illustrated, this creates an oversharpening at smooth transitions, which corresponds to the well-known 2D staircase effect. On the other hand, our proposed 2D regularization term yields better reconstruction performance (as in the 1D case) on piecewise-linear regions while preserving the edges satisfactorily.

As expected, the proposed 2D regularizer is thus welladapted for piecewise-linear image restoration. We assess our method quantitatively in Sec. 6 by performing deconvolution experiments on standard images.

\section{MINIMIZATION}

Our overall cost functional must be minimized according to (1). Since our regularizer is itself convex, as it is based on the spectral radius of the Hessian matrix, our overall cost is convex as well. Therefore, we resort to standard-descent optimization to find the solution, where the specific gradient expression related to our cost functional is derived below.

Since we are dealing with discrete data, the regularizer is discretized accordingly. Herein, we use vectors and matrices for discrete quantities, denoting them using bold notation; each vector corresponds to a lexicographically-ordered image sequence. Considering the discretized image $f$, and according to (4), we express the eigenvalues at each position $i$ as

$$
\lambda_{i}^{ \pm}=\left(\mathbf{T}_{1} \mathbf{f}\right)_{i} \pm \sqrt{\left(\mathbf{T}_{2} \mathbf{f}\right)_{i}^{2}+\left(\mathbf{T}_{3} \mathbf{f}\right)_{i}^{2}},
$$

where $\mathbf{T}_{1}, \mathbf{T}_{2}$, and $\mathbf{T}_{3}$ are block-circulant matrices implementing the discretized versions of the corresponding secondorder operations. Let use define $\lambda_{i}^{d}=\left|\lambda_{i}^{+}\right|-\left|\lambda_{i}^{-}\right|$. Then,

$$
\begin{aligned}
J_{\text {new }}\{\mathbf{f}\} & =\sum_{i} \max \left(\left|\lambda_{i}^{ \pm}\right|\right) \\
& =\sum_{i} \frac{1}{2}\left(\left|\lambda_{i}^{+}\right|+\left|\lambda_{i}^{-}\right|+\left|\lambda_{i}^{d}\right|\right) .
\end{aligned}
$$

For the sake of differentiability, the absolute value is approximated by a smooth Huber function [7]. The corresponding minimization problem is solved via the gradient-based algorithms [8]. The regularizer is differentiated as

$$
\begin{aligned}
\nabla J_{\text {new }}\{\mathbf{f}\} & =\frac{1}{2}\left[\mathbf{T}_{1}^{T} \mathbf{M}_{1} \mathbf{T}_{1}+\mathbf{T}_{2}^{T} \boldsymbol{\Theta} \mathbf{T}_{2}+\mathbf{T}_{3}^{T} \boldsymbol{\Theta} \mathbf{T}_{3}\right] \mathbf{f} \\
& +\frac{1}{2} \mathbf{T}_{1}^{T} \mathbf{M}_{2} \mathbf{g}
\end{aligned}
$$

where the vector $\mathbf{g}$ is defined by $\mathbf{g}_{i}=\sqrt{\left(\mathbf{T}_{1} \mathbf{f}\right)_{i}^{2}+\left(\mathbf{T}_{2} \mathbf{f}\right)_{i}^{2}}$, and where $\mathbf{M}_{1}, \mathbf{M}_{2}$, and $\Theta$ are the weighting diagonal matri-

\begin{tabular}{c|c|c|c|c}
\hline \hline & \multicolumn{2}{|c|}{ Uniform PSF } & \multicolumn{2}{c}{ Gaussian PSF } \\
\hline Image/ $\sigma$ & TV & Prop. & TV & Prop. \\
\hline Cell/0.56 & 7.07 & $\mathbf{7 . 4 6}$ & 6.09 & $\mathbf{6 . 4 4}$ \\
Cell/5 & 3.63 & $\mathbf{3 . 7 6}$ & 3.17 & $\mathbf{3 . 2 2}$ \\
Cell/7 & 3.75 & $\mathbf{3 . 8 1}$ & 3.45 & $\mathbf{3 . 5 3}$ \\
\hline Fing./0.56 & 9.59 & $\mathbf{9 . 9 3}$ & 8.67 & $\mathbf{8 . 8 5}$ \\
Fing./5 & 4.90 & $\mathbf{5 . 4 8}$ & 4.91 & $\mathbf{5 . 2 1}$ \\
Fing./7 & 4.37 & $\mathbf{4 . 8 2}$ & 4.42 & $\mathbf{4 . 7 8}$ \\
\hline Lena/0.56 & $\mathbf{7 . 1 5}$ & 7.05 & $\mathbf{6 . 1 2}$ & 5.94 \\
Lena/5 & $\mathbf{3 . 8 4}$ & 3.62 & $\mathbf{3 . 8 7}$ & 3.08 \\
Lena/7 & $\mathbf{3 . 8 2}$ & 3.54 & $\mathbf{3 . 5 1}$ & 3.17 \\
\hline \hline
\end{tabular}

Table 1. Restoration results for TV and our method with two different blurring kernels and three distinct noise levels.

ces

$$
\begin{gathered}
\mathbf{M}_{1}=\operatorname{diag}\left(\frac{1+\operatorname{sgn}\left(\lambda_{i}^{d}\right)}{\left|\lambda_{i}^{+}\right|}+\frac{1-\operatorname{sgn}\left(\lambda_{i}^{d}\right)}{\left|\lambda_{i}^{-}\right|}\right), \\
\mathbf{M}_{2}=\operatorname{diag}\left(\frac{1+\operatorname{sgn}\left(\lambda_{i}^{d}\right)}{\left|\lambda_{i}^{+}\right|}-\frac{1-\operatorname{sgn}\left(\lambda_{i}^{d}\right)}{\left|\lambda_{i}^{-}\right|}\right), \\
\mathbf{\Theta}=\operatorname{diag}\left(\frac{\operatorname{sgn}\left(\lambda_{i}^{+}\right)\left(1+\operatorname{sgn}\left(\lambda_{i}^{d}\right)\right)}{\mathbf{g}_{i}}\right. \\
\left.-\frac{\operatorname{sgn}\left(\lambda_{i}^{-}\right)\left(1-\operatorname{sgn}\left(\lambda_{i}^{d}\right)\right)}{\mathbf{g}_{i}}\right) .
\end{gathered}
$$

Finally, the gradient of the overall cost is given as

$$
\nabla J\{\mathbf{f}\}=-\mathbf{A}^{T}(\mathbf{y}-\mathbf{A f})+\frac{\lambda}{2} \nabla J_{n e w}\{\mathbf{f}\} .
$$

\section{EXPERIMENTAL RESULTS}

In this section, the performance of the proposed model is evaluated on standard natural images of size $512 \times 512$. We compare our proposed model with TV regularization. In our experiments, we consider two different types of blurring (Gaussian and moving average over a kernel of size $9 \times 9$ ). Further degradation is caused by Gaussian noise at distinct levels. The corresponding restoration results are reported in Table 1. For visual appreciation, two of these results are shown in Figs. 3 and 4. The results involving TV regularization are obtained using the available implementation of [1]. In all cases, the regularization parameter $\tau$ is fine-tuned to obtain the best performance in terms of the increase of signal-to-noise ratio (ISNR).

From Table 1, we see that our model performs better than TV for two of the considered images. Inspecting Figs. 3 and 4 , we observe that our model behaves better than TV in piecewise-linear transitions, which is in accordance with the properties of the regularizer discussed above. It also better preserves filament-like structures. For instance, the lower part of the Fingerprint image is relatively well restored, in contrast to TV which tends to merge or dilate the original structures. 


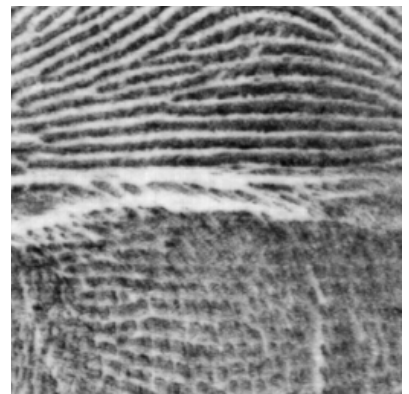

(a) Original image

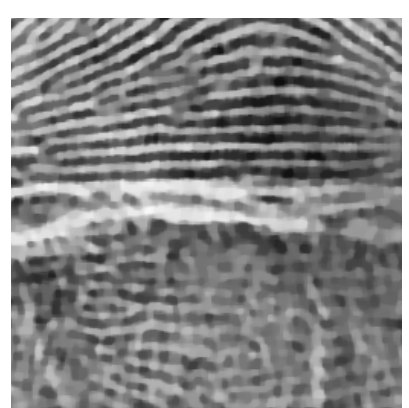

(c) TV solution $(\mathrm{ISNR}=4.90)$

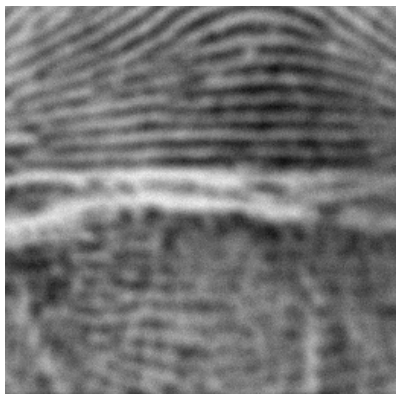

(b) Blurred and noisy image

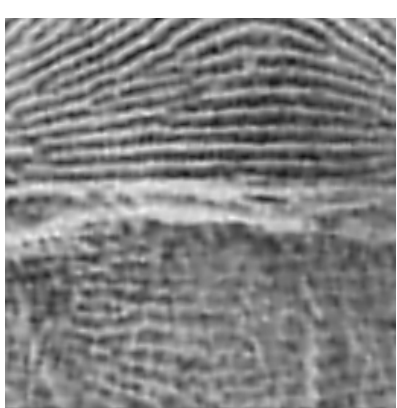

(d) Our solution $(\mathrm{ISNR}=5.21)$
Fig. 3. Close-up of the Fingerprint image for a Gaussian blurring kernel and a noise of $\sigma=5$.

\section{CONCLUSIONS}

We have proposed a second-order regularization method derived as an extension of TV. We have studied the potential applicability of our model, and compared its performance with respect to TV. The main advantage of our method is to allow for the reconstruction of piecewise-linear structure while preserving edges and ridges satisfactorily. Our experiments have shown the efficacy of our method for certain classes of images.

\section{REFERENCES}

[1] J.M. Bioucas-Dias, M.A.T. Figueiredo, and J.P. Oliveira, "Total variation-based image deconvolution: a majorization-minimization approach," in 2006 IEEE Int. Conf. Acoust., Speech and Signal Proc., May 2006, vol. 2, pp. 861-864.

[2] L. I. Rudin, S. Osher, and E. Fatemi, "Nonlinear total variation based noise removal algorithms," Phys. D, vol. 60, pp. 259-268, November 1992.

[3] A. Chambolle and P.-L. Lions, "Image recovery via total variation minimization and related problems," $\mathrm{Nu}$ merische Mathematik, vol. 76, pp. 167-188, 1997.

[4] T. Chan, A. Marquina, and P. Mulet, "High-order total

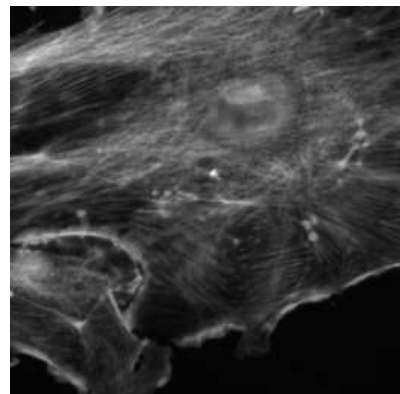

(a) Original image

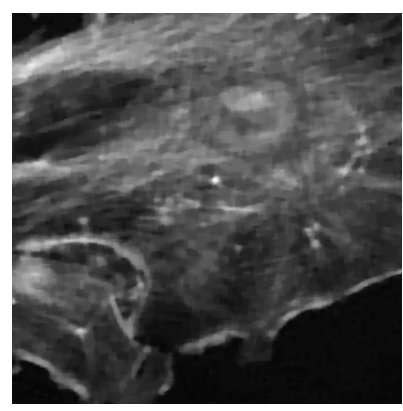

(c) TV solution $(\mathrm{ISNR}=6.09)$

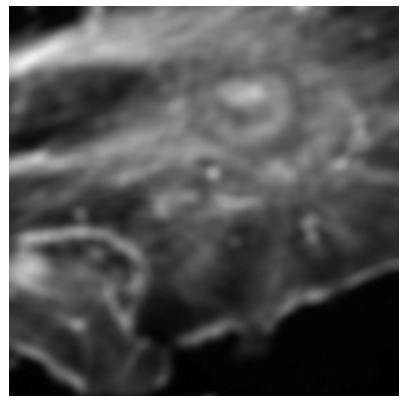

(b) Blurred and noisy image

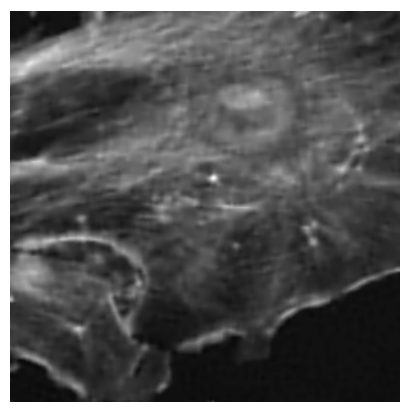

(d) Our solution $(\mathrm{ISNR}=6.44)$
Fig. 4. Close-up of the Cell image for a moving-average kernel and a noise of $\sigma=0.56$

variation-based image restoration," SIAM Journal on Scientific Computing, vol. 22, no. 2, pp. 503-516, 2000.

[5] G. Steidl, "A note on the dual treatment of higher order regularization functionals," Computing, vol. 76, pp. 135148, 2006.

[6] M. Lysaker, A. Lundervold, and X.-C. Tai, "Noise removal using fourth-order partial differential equations with applications to medical magnetic resonance images in space and time," IEEE Trans. Imag. Proc, vol. 12, pp. 1579-1590, 2003.

[7] M. Nikolova and K. Michael, "Analysis of half-quadratic minimization methods for signal and image recovery," SIAM Journal of Scientific Computing, vol. 27, no. 3, pp. 937-966, 2006.

[8] S. Boyd and L. Vandenberghe, Convex Optimization, Kluwer Academic Publishers, Massachusetts,USA, March 2004. 